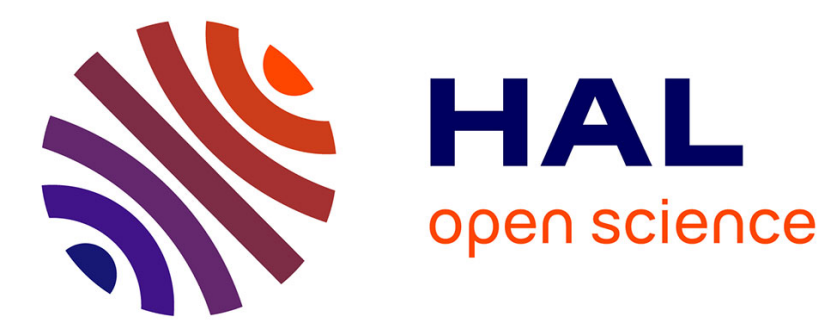

\title{
A Distributed-Feedback Diode Laser Frequency Stabilized on Doppler-Free Cs D1 Line
}

\author{
X. Liu, R. Boudot
}

\section{To cite this version:}

X. Liu, R. Boudot. A Distributed-Feedback Diode Laser Frequency Stabilized on Doppler-Free Cs D1 Line. IEEE Transactions on Instrumentation and Measurement, 2012, 61 (10), pp.2852-2855. 10.1109/TIM.2012.2196399 . hal-00744542

\section{HAL Id: hal-00744542 \\ https://hal.science/hal-00744542}

Submitted on 30 Apr 2021

HAL is a multi-disciplinary open access archive for the deposit and dissemination of scientific research documents, whether they are published or not. The documents may come from teaching and research institutions in France or abroad, or from public or private research centers.
L'archive ouverte pluridisciplinaire HAL, est destinée au dépôt et à la diffusion de documents scientifiques de niveau recherche, publiés ou non, émanant des établissements d'enseignement et de recherche français ou étrangers, des laboratoires publics ou privés. 


\title{
A Distributed-Feedback Diode Laser Frequency Stabilized on Doppler-Free Cs $D_{1}$ Line
}

\author{
Xiaochi Liu and Rodolphe Boudot
}

\begin{abstract}
This paper describes a distributed-feedback laser diode system frequency stabilized by simple saturated absorption technique at $895 \mathrm{~nm}$ on the Cs $D_{1}$ line with a linewidth narrower than $1 \mathrm{MHz}$. This laser source was developed to be used in a compact cesium atomic clock based on coherent population trapping. The frequency stability of the laser system, evaluated by measuring the beat note of two similar laser systems and characterized by Allan deviation, is measured to be lower than $1 \times 10^{-11}$ for integration times of up to $2000 \mathrm{~s}$, even achieving $4 \times 10^{12}$ at $200 \mathrm{~s}$. This laser diode system, mounted in a mechanically stable cage system using easy-to-find commercially available components, remains in locked operation for several weeks without any problem.
\end{abstract}

Index Terms-Allan deviation, distributed-feedback laser, laser frequency stabilization, saturated absorption technique.

\section{INTRODUCTION}

$\mathbf{F}$ REQUENCY-STABILIZED laser diode systems are required and applied variously in different fields for metrological purposes and experiments such as atomic-frequency standards, atomic magnetometers, laser-cooling experiments, high-resolution spectroscopy, coherent optical communications, or accurate geophysical measurements using laser interferometers. Numerous techniques are described in the literature for laser wavelength stabilization [1]. Fast frequency-noise reduction of a laser system is generally achieved by locking it onto the transmission peak of a reference ultrastable FabryPerot cavity using the Pound-Drever-Hall technique [2]. A continuous wave laser stabilized to such a cavity can achieve a subhertz linewidth and a fractional frequency instability as low as $2 \times 10^{-16}$ for averaging times of $1-10 \mathrm{~s}$ [3]-[6]. A compact alternative method to cavity stabilization for fast Fourier frequency-noise reduction consists to use an optical fiber delay line interferometer with arm imbalance to measure the laser frequency fluctuations during a fixed time delay [7]-[9]. When long-term operation and stability are required, laser frequency stabilization by comparison to an atomic or molecular absorption line is preferred. Best performances are generally obtained

This work was supported by Laboratoire National de Métrologie et d'Essais (LNE) under Scientific Program Contract LNE-DRST 10-3-005. The work of $\mathrm{X}$. Liu was supported by Région de Franche-Comté. The Associate Editor coordinating the review process for this paper was Dr. George Xiao.

The authors are with Franche-Comté Electronique Mecanique Thermique Optique-Sciences et Technologies (FEMTO-ST), Centre National de la Recherche Scientifique (CNRS), 25044 Besancon, France (e-mail: rodolphe.boudot@femto-st.fr; xiaochi.liu@femto-st.fr). by combining saturated absorption techniques to reduce acoustic noise and narrow-linewidth $(\sim 100 \mathrm{kHz})$ extended-cavity diode lasers (ECDLs) [10]-[12]. Interference-filter-stabilized external-cavity diode lasers have even demonstrated best potential and performances [13] than classical diffraction-grating wavelength selection. Nevertheless, stable operation of ECDL requires high immunity to ambient vibrations, optical misalignment of the optical feedback path, accurate control of the cavity length, and, consequently, multiple feedback servo paths for wavelength stabilization.

Due to progress in the technology of semiconductor lasers and low-frequency-noise levels, distributed feedback (DFB) and distributed Bragg resonator (DBR) lasers now represent a potential cost-effective alternative when a narrow linewidth is desired. DBR lasers suffer from periodic nonlinearities in the light-current characteristics due to longitudinal mode hopping. In contrast, DFB lasers can operate in the same longitudinal mode over a large current range and exhibit a larger side-mode suppression. DFB linewidths greatly lower than $1 \mathrm{MHz}$ have already been demonstrated [14], even exhibiting linewidths as narrow as $350 \mathrm{kHz}$ [15] at $895 \mathrm{~nm}$. For Cs atom physics experiments, most of the literature refers to laser frequency stabilization on the $\mathrm{Cs} D_{2}$ line at $852 \mathrm{~nm}$. Typical reported fractional frequency stability is found to be from $4 \times 10^{-13}$ to a few $10^{-12}$ at 1-s integration time with ECDLs [10], while it is measured to be $1 \times 10^{-11}$ [16] $-1 \times 10^{-10}$ with DBR diode lasers [17].

This paper reports on frequency stabilization of a commercially available DFB diode laser system onto the Cs $D_{1}$ line at $895 \mathrm{~nm}$ using standard saturated absorption method. This wavelength was selected because our laser is devoted to be used in a coherent population trapping (CPT) Cs vapor cell clock [18]. To our knowledge, typical laser frequency stability performances obtained by stabilization against Cs Doppler-free $D_{1}$ line at $895 \mathrm{~nm}$ have never been clearly reported.

\section{DFB LASER SYSTEM}

Fig. 1 reports the DFB laser system combined with the saturated absorption scheme.

The laser source is a commercially available GaAs semiconductor DFB laser diode with integrated grating structure emitting in a single mode at $895 \mathrm{~nm}$. It is housed in a hermetic TO-3 package with thermistance and thermoelectric cooler. The maximum output optical power is about $50 \mathrm{~mW}$, while its threshold current is about $50 \mathrm{~mA}$. The temperature coefficient of wavelength is about $0.06 \mathrm{~nm} / \mathrm{K}$, while the dependence of wavelength on injection current is $0.003 \mathrm{~nm} / \mathrm{mA}$. The diode laser is driven by a low-noise current controller inspired from [19] exhibiting a current noise power spectral density lower than $50 \mathrm{pA} / \sqrt{\mathrm{Hz}}$ 


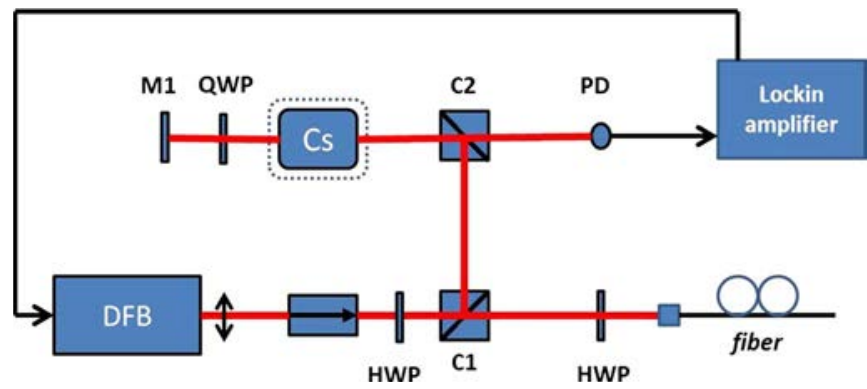

Fig. 1. DFB laser diode optical setup. (QWP) Quarter-wave plate. (HWP) Half-wave plate. (PD) Photodiode. (M1) Mirror. (C1 and C2) Polarizing cube splitters. (Cs) Cs cell.

in a $1-\mathrm{kHz}$ bandwidth. The diode laser temperature is stabilized within the millikelvin level with a high-precision temperature controller using the internal Peltier cooler.

The output beam of the DFB laser is collimated using an antireflection-coated aspheric lens with a focal length of $2.5 \mathrm{~mm}$ and a numerical aperture of 0.55 . The output of the DFB laser is passed through an optical Faraday isolator $(45 \mathrm{~dB})$ in order to avoid optical feedback. A polarizing beam splitter cube (C1) combined with a half-wave plate is then used to split the optical beam in two directions.

The first beam is directed to the saturated absorption setup used to stabilize the laser frequency. In the first sense, the 3-mm-diameter beam is used as the pump beam to saturate cesium vapor in a 2-cm-diameter and 2.5-cm-long antireflection-coated glass cell. At the output of the cell, the light is retroreflected by a silver-coated mirror and used as the probe beam. The reference cell is surrounded by a singlelayer mu-metal magnetic shield in order to avoid perturbations from environmental magnetic fields. For optimized long-term operation and stability, the cell is placed in an oven carefully temperature stabilized using an improved analog-digital version of the electronic temperature controller described in [20]. The cell temperature sensor is controlled below the millikelvin level at 1 -s integration time and better than $10^{-5}$ level at $4000 \mathrm{~s}$. The light is detected at the output of the cube splitter $\mathrm{C} 2$ by a low-noise photodiode followed by a transimpedance amplifier.

The laser frequency stabilization is achieved by applying a low-frequency sinusoidal modulation $(60 \mathrm{kHz})$ added to the DFB laser injection current. The output voltage from the photodiode absorption signal is amplified, filtered, and synchronously demodulated using a laboratory-prototype digital lock-in amplifier. The latter provides a dispersive error signal used to adjust the laser frequency by correcting its injection current through a proportional-integral controller.

The second output beam from the cube $\mathrm{C} 1$, used as the main output for the Cs CPT clock experiment, is sent through a second half-wave plate and potentially injected in a pigtailed fiber-optic connector-angled physical contact (FC-APC) optical fiber using a fiber port collimation system. The DFB laser system ensemble is mounted in a compact mechanically stable cage system using commercially available components and isolated from acoustic vibrations by a sophisticated optical table with pneumatic damping.

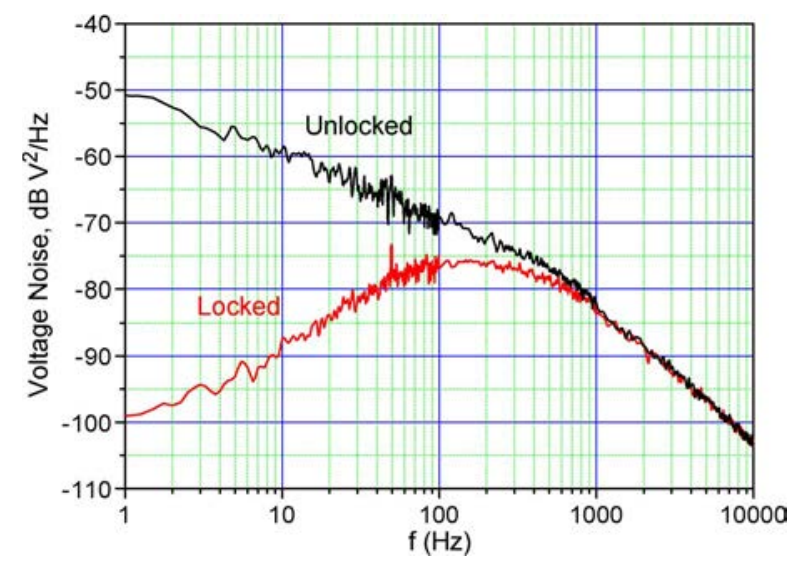

Fig. 2. Voltage noise of the laser frequency servo lock-in amplifier output (servo on or servo off).

\section{EXPERIMENTAL RESUlts}

Fig. 2 reports the voltage noise of the laser frequency servo lock-in amplifier output in free-running and locked conditions. Optimal conditions result from a tradeoff between loop noise reduction for long-term operation and control bandwidth. In this case, the laser frequency servo bandwidth is about $400 \mathrm{~Hz}$, while the noise level at $1-\mathrm{Hz}$ offset frequency is reduced by about $45 \mathrm{~dB}$.

Two laser systems similar to the one described in Section II were built to measure the typical DFB beat-note linewidth and frequency stability as shown in Fig. 3. Both laser systems use independent reference cells. The first DFB laser is frequency stabilized onto the Cs $4-3^{\prime}$ transition, while the second is locked onto the Cs $4-4^{\prime}$ transition. The frequency difference between these two transitions is about $1.16 \mathrm{GHz}$. For linewidth measurement, the main output beam from both lasers is injected in a 50-50-splitting-ratio optical polarization-maintaining fiber coupler. The coupler is used to mix the two beams and generate the beat-note signal. FC-APC fiber connectors are used to avoid optical feedback. The beat-note signal is detected by a fast photodiode, amplified by $23 \mathrm{~dB}$ with a low-noise amplifier, low-pass filtered, and analyzed with a microwave spectrum analyzer.

Fig. 4 gives the spectrum of the beat-note signal at $1.16 \mathrm{GHz}$ from two similar DFB laser systems in locked configuration. The full-width at half-maximum (FWHM) of the beat note is measured to be $1.95 \mathrm{MHz}$ in free-running conditions. The linewidth of a single laser is about $975 \mathrm{kHz}$.

For laser frequency stability measurement (see Fig. 3), the resulting $1.16-\mathrm{GHz}$ beat-note signal is compared to a $1.16 \mathrm{GHz}+6 \mathrm{MHz}$ signal coming from an IFR2023A synthesizer driven by a hydrogen maser. The final beat note at $6 \mathrm{MHz}$ is low-pass filtered, amplified, and counted using a highresolution frequency counter. This frequency down-conversion to $6 \mathrm{MHz}$ was done not only because no microwave counter was available in the laboratory but also to improve the frequencycounting resolution.

Fig. 5 shows the Allan deviation versus the integration time of the beat-note signal frequency under free-running or locked conditions. 


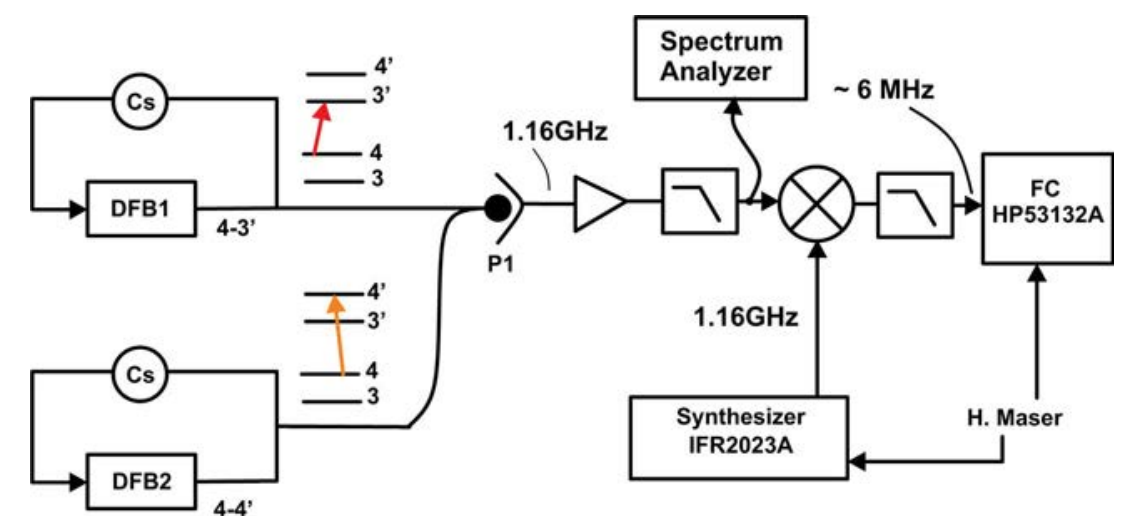

Fig. 3. DFB optical beat note and frequency stability experimental setup.

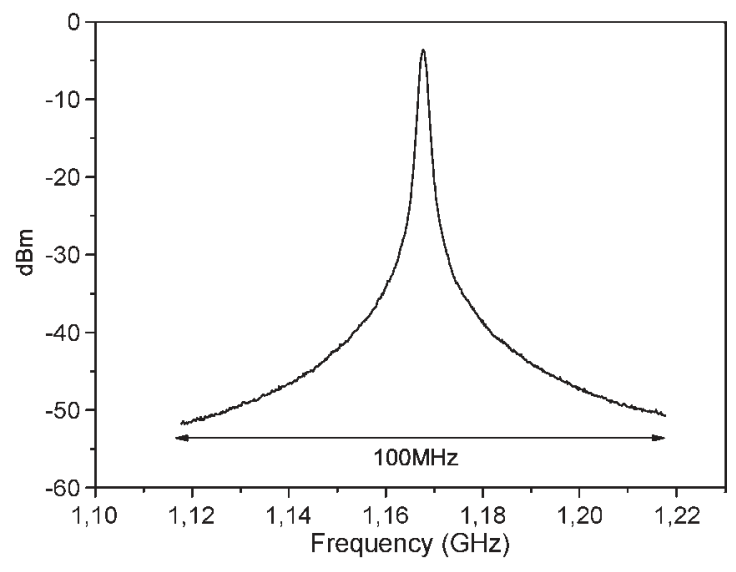

Fig. 4. DFB beat-note spectrum in locked configuration. The spectrum analyzer resolution bandwidth is set to $30 \mathrm{kHz}$. The FWHM of a single laser is $975 \mathrm{kHz}$ in free-running conditions.

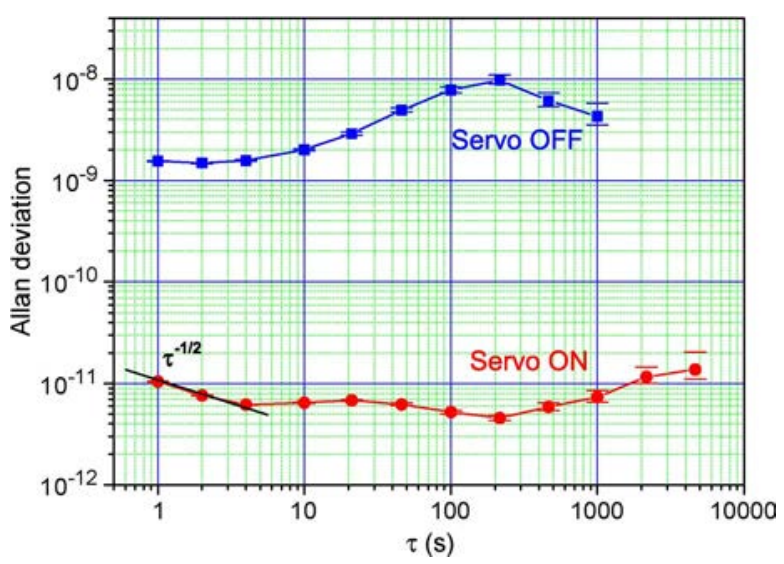

Fig. 5. Allan deviation of the DFB laser beat-note frequency (servo on or servo off)

In the free-running regime, the short-term frequency stability is measured to be $1.5 \times 10^{-9}$ at 1 -s integration time, increasing to reach about $8 \times 10^{-9}$ at 100 -s integration time. In the stabilized regime, the laser beat note exhibits a frequency instability of $1 \times 10^{-11}$ at 1 -s integration time and decreases with a $\sqrt{\tau}$ slope until $4 \mathrm{~s}$ before reaching a wide flat flicker floor at the level of about $4-5 \times 10^{-12}$. For averaging times longer than $200 \mathrm{~s}$, the Allan deviation increases again without showing a clear $\tau$ slope drift. These results compare favorably with those presented in [16] and [17] on the Cs $D_{2}$ line. We are confident that the laser system remains locked for several weeks without any problem.

Frequency stability performances of our DFB laser system are greatly satisfying for a CPT Cs vapor cell clock application. In such a clock, laser frequency variations can induce a clock frequency variation through the light-shift-induced effect. Typical reported values are a clock frequency variation of about $-20 \mathrm{mHz}$ for a 1-MHz laser frequency change [21]. In our setup, the DFB laser frequency instability of $1 \times 10^{-11}$ corresponds to a typical frequency variation of $3.5 \mathrm{kHz}$. The clock frequency stability limitation due to laser frequency changes is consequently reduced to a level of $7.6 \times 10^{-15}$ that is several orders of magnitude lower than frequency stability performances obtained with the state-of-the-art CPT-based Cs vapor cell atomic-frequency references [22].

\section{CONCLUSION}

We have demonstrated a DFB laser system frequency stabilized by classical saturated absorption technique on the $\mathrm{Cs}$ Doppler-free $D_{1}$ line at $895 \mathrm{~nm}$. It exhibits a fractional frequency instability better than $10^{-11}$ at 1 -s integration time and lower than $5 \times 10^{-12}$ at $200 \mathrm{~s}$. This laser source will be used in a Cs vapor cell clock based on CPT.

\section{ACKNOWLEDGMENT}

The authors would like to thank C. Rocher and P. Abbé for technical support and electronics design.

\section{REFERENCES}

[1] W. Demtröder, Laser Spectroscopy: Basic Concepts and Instrumentation-Third Edition., 3rd ed. New York: Springer-Verlag, 2002, sec. 5.4.5.

[2] R. W. P. Drever, J. L. Hall, F. V. Kowalski, J. Hough, G. M. Ford, A. J. Munely, and H. Ward, "Laser phase and frequency noise stabilization using an optical resonator," Appl. Phys. B, vol. 31, no. 2, pp. 97-105, 1983.

[3] A. D. Ludlow, X. Huang, M. Notcutt, T. Zanon-Wilette, S. M. Foremand, M. M. Boyd, S. Blatt, and J. Ye, "Compact thermal-noise-limited optical cavity for diode laser stabilization at $1 \times 10^{-15}$," Opt. Lett., vol. 32, no. 6, pp. 641-643, Mar. 2007.

[4] S. A. Webster, M. Oxborrow, S. Pugla, J. Millo, and P. Gill, "Thermalnoise-limited optical cavity," Phys. Rev. A, vol. 77, no. 3, p. 033847, Mar. 2008.

[5] J. Millo, D. V. Magalahes, C. Manddache, Y. Le Coq, E. M. L. Ensglish, P. W. Westergaard, J. Lodewyck, S. Bize, P. Lemonde, and G. Santarelli, 
"Ultra-stable lasers based on vibration insensitive cavities," Phys. Rev. A, vol. 79, no. 5, pp. 053829-1-053829-7, May 2009.

[6] Y. Y. Jiang, A. D. Ludlow, N. D. Lemke, R. W. Fox, J. A. Sherman, L. S. Ma, and C. W. Oates, "Making optical atomic clocks more stable with $10^{-16}$ level laser stabilization," Nature Photon., vol. 5, pp. 158161, 2011.

[7] G. A. Cranch, "Frequency noise reduction in erbium-doped fiber distributed-feedback lasers by electronic feedback," Opt. Lett., vol. 27, no. 13, pp. 1114-1116, Jul. 2002.

[8] F. Kefelian, H. Jiang, P. Lemonde, and G. Santarelli, "Ultra-low frequency-noise stabilization of a laser by locking to an optical fiber-delay line," Opt. Lett., vol. 34, no. 7, pp. 914-916, 2009.

[9] W. K. Lee, C. Y. Park, J. Mun, and D. H. Yu, "Linewidth reduction of a distributed-feedback diode laser using an all-fiber interferometer with short path imbalance," Rev. Sci. Instrum., vol. 82, no. 7, pp. 073105-1073105-5, Jul. 2011.

[10] G. D. Rovera, G. Santarelli, and A. Clairon, "A laser diode system stabilized on the caesium $D_{2}$ line," Rev. Sci. Instrum., vol. 65, no. 5, pp. 15021505, Mar. 1994.

[11] C. Affolderbach and G. Mileti, "Tuneable, stabilized diode lasers for compact atomic frequency standards and precision wavelength references," Opt. Lasers Eng., vol. 43, no. 3-5, pp. 291-302, 2005.

[12] C. Affolderbach and G. Mileti, "A compact laser head with highfrequency stability for Rb atomic clocks and optical instrumentation," Rev. Sci. Instrum., vol. 76, no. 7, pp. 073108-1-073108-5, Jul. 2005.

[13] X. Baillard, A. Gauguet, S. Bize, P. Lemonde, P. Laurent, A. Clairon, and P. Rosenbusch, "Interference-filter-stabilized external-cavity diode lasers," Opt. Comm., vol. 266, no. 2, pp. 609-613, Oct. 2006.

[14] F. J. Vermersch, V. Ligeret, S. Bansropun, M. Lecomte, O. Parillaud, M. Calligaro, M. Krakowski, and G. Giuliani, "High-power narrow linewidth distributed feedback lasers with an aluminium-free active region emitting at $852 \mathrm{~nm}$," IEEE Photon. Technol. Lett., vol. 20, no. 13, pp. 1145-1147, Jul. 2008

[15] A. Klehr, H. Wenzel, O. Brox, F. Bugge, G. Erbert, T. P. Nguyen, and G. Trankle, "High-power 894 nm monolithic distributed-feedback laser," Opt. Exp., vol. 15, no. 18, pp. 11364-11369, Sep. 2006.

[16] F. Bertinetto, P. Cordiale, G. Galzerano, and E. Bava, "Frequency stabilization of DBR diode laser against Cs absorption lines at $852 \mathrm{~nm}$ using the modulation transfer method," IEEE Trans. Instrum. Meas., vol. 50, no. 2, pp. 490-492, Apr. 2001.

[17] T. Hori, A. Araya, S. Moriwaki, and N. Mio, "Development of a wavelength-stabilized distributed Bragg reflector laser diode to the Cs $\mathrm{D}_{2}$ line for field use in accurate geophysical measurements," Rev. Sci. Instrum., vol. 78, no. 2, pp. 026105-1-026105-3, Feb. 2007.
[18] M. Stahler, R. Wynands, S. Knappe, J. Kitching, L. Hollberg, A. Taichenachev, and V. Yudin, "Coherent population trapping resonances in thermal ${ }^{85} \mathrm{Rb}$ vapor: $D_{1}$ versus $D_{2}$ line excitation," Opt. Lett., vol. 27 , no. 16 , pp. $1472-1474$, Aug. 2002

[19] K. G. Libbrecht and J. L. Hall, "A low-noise high-speed diode laser current controller," Rev. Sci. Instrum., vol. 64, no. 8, pp. 2133-2135, Aug. 1993.

[20] R. Boudot, C. Rocher, N. Bazin, S. Galliou, and V. Giordano, "Highprecision temperature stabilization for sapphire resonators in microwave oscillators," Rev. Sci. Instrum., vol. 76, no. 9, pp. 095110-1-095110-6, Sep. 2005.

[21] S. Knappe, R. Wynands, J. Kitching, H. G. Robinson, and L. Hollberg, "Characterization of coherent population trapping resonances as atomic frequency references," J. Opt. Soc. Amer. B, vol. 18, no. 11, pp. 15451553, Nov. 2001.

[22] R. Boudot, S. Guerandel, E. De Clercq, N. Dimarcq, and A. Clairon, "Current status of a pulsed CPT Cs cell clock," IEEE Trans. Instrum. Meas., vol. 58, no. 4, pp. 1217-1222, Apr. 2009. 